\title{
Expression and activity of the TLR4/NF-кB signaling pathway in mouse intestine following administration of a short-term high-fat diet
}

\author{
NING WANG ${ }^{1}$, HUGUO WANG $^{2}$, HUA YAO $^{3}$, QIN WEI $^{4}$, XIN-MIN MAO 5 , \\ TAO JIANG ${ }^{4}$, JING XIANG ${ }^{6}$ and NA DILA ${ }^{1}$
}

\begin{abstract}
Departments of ${ }^{1}$ No. 1 Cadre Wards Medicine, ${ }^{2}$ Digestive and Vascular Surgery, ${ }^{3}$ Metabolic Disease and
${ }^{4}$ Biomedical Sciences, First Affiliated Hospital of Xinjiang Medical University, Urumqi, Xinjiang 830011;

${ }^{5}$ Experimental Teaching Demonstration Centre, Pharmaceutical College of Xinjiang Medical University, Urumqi, Xinjiang 830011; ${ }^{6}$ Department of Endocrinology, Xinjiang Petroleum Administration

Bureau General Hospital, Urumqi, Xinjiang 830011, P.R. China
\end{abstract}

Received February 13, 2013; Accepted May 10, 2013

DOI: $10.3892 /$ etm.2013.1214

\begin{abstract}
Insulin resistance in obesity is associated with chronic systemic low-grade inflammation. Although it has been shown that Toll-like receptor 4 (TLR4) in the liver, muscle and adipose tissue plays an important role in obesity-associated inflammation and insulin resistance, the effect of TLR4 activation in the intestine has not been investigated. The aim of this study was to explore the activation of the mouse intestinal TLR4/NF- $\kappa$ B signaling pathway following the administration of a short-term high-fat diet, as well as the function of the signaling pathway in the local enteric inflammatory response. The effect of the high-fat diet on TLR4 activation, NF- $\kappa \mathrm{B}$ and phosphorylated $\mathrm{I} \kappa \mathrm{B}(\mathrm{PI} \mathrm{B})$ activity, and tumor necrosis factor (TNF)- $\alpha$ and IL- 6 expression in the intestinal tissues of diet-induced obese C57BL/6 mice was investigated. The results demonstrated that the high-fat diet induced TLR4 mRNA and protein expression in intestinal tissues. TLR4/NF- $\kappa \mathrm{B}$ signaling pathway activation gradually increased as the number of days of high-fat diet administration increased, and peaked on day 7 . Additionally, activation of the signaling pathway reduced PI $\kappa \mathrm{B}$ expression levels and increased TNF- $\alpha$ and IL-6 expression levels in intestinal tissues. Our results demonstrated that a short-term high-fat diet induces activation of the TLR4/NF- $\kappa$ B signaling pathway in intestinal tissues, which causes local intestinal low-grade inflammation. These data improve our understanding of the molecular events involved in intestinal
\end{abstract}

Correspondence to: Dr Ning Wang, Department of No. 1 Cadre Wards Medicine, First Affiliated Hospital of Xinjiang Medical University, 137 Carp Mount Road, Urumqi, Xinjiang 830011, P.R. China

E-mail: ningwangcn@126.com

Key words: Toll-like receptor 4/NF-kB, short-term high-fat diet, intestines, local inflammatory response low-grade inflammation, which may be the triggering factor for chronic systemic low-grade inflammation.

\section{Introduction}

A great deal of attention has been paid to the theory of inflammation and certain researchers consider that diabetes, obesity and atherosclerosis represent a low-grade, chronic inflammatory condition. Pickup (1) hypothesized that stimulation from dietary surplus and other environmental factors results in the activation of a specific cell population of the innate immune system. Certain sentinel cells, including macrophages and fat cells, secrete tumor necrosis factor (TNF)- $\alpha$, IL-6 and other inflammatory agents that cause a low-grade inflammatory condition and accordingly trigger insulin resistance, diabetes and related diseases. Toll-like receptor 4 (TLR4) is one of the receptors that is able to identify pathogenic microorganisms in a natural immune system, bind the specific ligand and produce corresponding inflammation following identification. One study identified that TLR4 is related to whole-body, low-grade chronic inflammatory diseases, including those mentioned above (2).

Previous studies have demonstrated that TLR4 may be a central link between insulin resistance, inflammation and obesity, and that a point mutation in TLR4, which inactivates the receptor, prevents the diet-induced obesity (DIO) activation of I $\kappa \mathrm{B}$ kinase $(\mathrm{IKK} \beta)$ and c-Jun NH2-terminal kinase (JNK), and inhibits insulin resistance, suggesting that TLR4 is a key modulator in the cross-talk between inflammatory and metabolic pathways (3-8).

TLR4 exogenous ligands include lipopolysaccharides (LPS), endogenous ligands, including free fatty acids (FFA) (9), high-molecular-weight sugar (10) and the surface of fungi polysaccharides (10). Myenteric neurons mediate LPS recognition via TLR4, resulting in neuronal cell death (11). Continuous FFA stimulation activates TLR4 signaling pathways; however, when TLR4 is blocked or knocked out, FFA stimulation is also blocked (12). In addition, triglycerides (TG) 
also activate TLR4/NF- $\mathrm{KB}$ and increase the expression of inflammatory cytokines. One study demonstrated that acute and chronic, physical exercise in DIO rats induces significant suppression in the TLR4 signaling pathway in the liver, muscle and adipose tissue, reduces LPS serum levels and improves insulin signaling and sensitivity (13). Therefore, low-grade inflammation triggered by a high-fat diet is closely associated with TLR4 expression. The main focal point of current research is TLR4 receptors in insulin-sensitive organs and tissues, including fat, liver, pancreas and skeletal muscle; however, studies concerning intestinal TLR4 have not yet been reported.

The intestines are not only primary immune organs, but they also have the most extensive surface area compared with other immune organs. The TLR4 receptors, which are distributed on the intestinal surface, recognize enteric pathogen-associated molecular patterns (PAMPs) and activate NF- $\mathrm{kB}$. Then, signals incorporated into the corresponding promoter and enhancer DNA binding sites in the cytokine gene regulation area initiate gene transcription and expression (14). Induced cytokines further activate immunocytes or cytokine receptors, expand the immune reaction, cause excessive release of inflammatory mediators and produce a cascade reaction of inflammatory factors. As a sensor of endogenous lipid and fatty acids, TLR4 regulates metabolism and the immune system (15).

On the basis of data from previous studies, we hypothesize that intestinal TLR4, activated by components of the high-fat diet, may become a key trigger for intestinal low-grade inflammation. Thus, the present study aimed to analyze the effect of mouse intestinal TLR4/NF- $\mathrm{kB}$ signaling pathway activation caused by a short-term, high-fat diet, as well as the function of the signaling pathway in the local enteric inflammatory response.

\section{Materials and methods}

Animals. Adult male C57BL/6 mice $(\mathrm{n}=60)$ aged 6 weeks, weighing $18 \pm 2 \mathrm{~g}$, were purchased from Xinjiang Medical University Animal Experimental Center (Urumqi, China). All animals were housed and used in accordance with the Chinese Regulations for Animal Care. This study was performed in strict accordance with the recommendations in the Guide for the Care and Use of Laboratory Animals of the National Institutes of Health. The animal use protocol was reviewed and approved by the Institutional Animal Care and Use Committee (IACUC) of the First Affiliated Hospital of Xinjiang Medical University. All experiments were conducted with the approval of the animal ethics committees of Xinjiang Medical University. The mice were randomly divided into six groups and each group of ten mice was fed with a high-fat diet for $0,1,3,5,7$ and 9 days, respectively. The formula of the high-fat diet was added to common fodder as follows: $10 \%$ sucrose, $28 \%$ maltodextrin, $0.12 \%$ choline chloride, $10 \%$ lard, $27.5 \%$ casein, $0.24 \%$ methionine and $0.1 \%$ sodium chloride.

Hematoxylin and eosin $(H \& E)$ staining. The mice were sacrificed by decapitation after $6 \mathrm{~h}$ of fasting in accordance with experimental requirements. The intestines were removed, absterged with physiological saline solution and then stored at $-80^{\circ} \mathrm{C}$ until analysis.
A $1 \mathrm{~cm}$ length of intestinal tissue from each sample was obtained. A microscope slide with rehydrated tissue sections was fixed in alcohol. The slide was immersed for $30 \mathrm{sec}$ with agitation by hand in $\mathrm{H}_{2} \mathrm{O}$. The slide was dipped into a Coplin jar containing Mayer's hematoxylin and agitated for $30 \mathrm{sec}$. The slide was rinsed with $\mathrm{H}_{2} \mathrm{O}$ for $1 \mathrm{~min}$. The slide was stained with $1 \%$ eosin $\mathrm{Y}$ solution for $10-30 \mathrm{sec}$ with agitation. The sections were dehydrated with two changes of $95 \%$ alcohol and two changes of $100 \%$ alcohol for $30 \mathrm{sec}$ each. The alcohol was removed with two changes of xylene. One or two drops of mounting medium was added and the section was covered with a coverslip. The intestinal mucosa was observed by light microscopy using the single-blind method.

Immunohistochemistry. Intestinal tissue was fixed with paraformalin $(40 \mathrm{~g} / \mathrm{l})$, embedded in paraffin, sectioned using a microtome $(4 \mu \mathrm{m})$, deparaffinized by xylene, dehydrated with a graded alcohol series, blocked with $10 \%$ goat serum for $30 \mathrm{~min}$ at room temperature in order to block nonspecific binding and repaired. TNF- $\alpha$ and IL- 6 were detected using rabbit anti-TNF- $\alpha$ and anti-IL-6 polyclonal antibodies (Abs), followed by application of horseradish peroxidase (HRP)labeled goat secondary Abs. The presence of brown staining in the cytoplasm and/or nuclei indicated that cells were positive for TNF- $\alpha$ or IL-6. Each sample was randomly assessed with five dyes at high magnification (x400). Positive cells were graded and scored according to a coloring scale: no coloring $(-, 0$ points), coloring area $<25 \%$ (,+ 1 point), coloring area $25-50 \%(++, 2$ points) and coloring area $>50 \%(+++, 3$ points).

Western blot analysis. Briefly, holoprotein extracts $(70 \mathrm{mg})$ of the mouse intestinal tissue in each sample were electrophoresed on a 10\% sodium dodecyl sulfate (SDS)-polyacrylamide gel and transferred to polyvinylidene fluoride (PVDF) membranes. Activated TLR4, NF- $\kappa \mathrm{B}$ and phosphorylated IкB (РІкB) were detected using rabbit Abs. Membranes were blocked and incubated with appropriate $\mathrm{Abs}$ at $4^{\circ} \mathrm{C}$ overnight, then imaged by conjugation with a HRP-linked secondary antibody and enhanced chemiluminescence (ECL) detection reagent. All experiments were performed in triplicate with similar results. All protein expression was divided by the amount of $\beta$-actin of individual samples as analyzed by image software. The blots were analyzed by densitometry.

Real-time polymerase chain reaction (PCR). The mRNA expression was analyzed using reverse transcription (RT)PCR. Total RNA from mouse intestines was extracted using TRIzol reagent (Invitrogen Life Technologies, Carlsbad, CA, USA). Each RNA sample ( $5 \mu \mathrm{g})$ was diluted and reverse-transcribed into complementary DNA (cDNA), to provide transcripts $(3 \mu \mathrm{g})$ for amplification. The primer sequences for amplification of the cDNA were as follows: TLR4, forward: 5'-CACTGTTCTTCTCCTGCCTGAC-3' and reverse, 5'-TGG TTGAAGAAGGAATGTCATC-3'); NF- $\kappa B$, forward: 5'-CCT CTGGCGAATGGCTTTAC-3' and reverse: 5'-GCTATGGAT ACTGCGGTCTGG-3'; $\beta$-actin, forward: 5'-CACGATGGA GGGGCCGGACTCATC-3' andreverse:5'-TAAAGACCTCTA TGCCAACACAGT-3'). The PCR conditions were as follows: i) $95^{\circ} \mathrm{C}$ for $2 \mathrm{~min}$ for one cycle; ii) $95^{\circ} \mathrm{C}$ for $45 \mathrm{sec}$; iii) $54^{\circ} \mathrm{C}$ for $45 \mathrm{sec}$; iv) $72^{\circ} \mathrm{C}$ for $1 \mathrm{~min}$ (modified for each primer set); 
Table I. Distribution of TNF- $\alpha$ in mouse intestines.

\begin{tabular}{lllllll}
\hline & \multicolumn{5}{c}{ Time (days) } \\
\cline { 2 - 7 } Score & 0 & 1 & 3 & 5 & 7 & 9 \\
\hline- & 6 & 5 & 4 & 2 & 0 & 0 \\
+ & 0 & 1 & 2 & 4 & 4 & 2 \\
++ & 0 & 0 & 0 & 0 & 2 & 2 \\
+++ & 0 & 0 & 0 & 0 & 0 & 2
\end{tabular}

TNF, tumor necrosis factor.

Table II. Distribution of IL-6 in mouse intestines

\begin{tabular}{lllllll}
\hline & \multicolumn{7}{c}{ Times (days) } \\
\cline { 2 - 7 } Score & 0 & 1 & 3 & 5 & 7 & 9 \\
\hline- & 6 & 5 & 4 & 2 & 1 & 1 \\
+ & 0 & 1 & 2 & 3 & 3 & 2 \\
++ & 0 & 0 & 0 & 1 & 2 & 2 \\
+++ & 0 & 0 & 0 & 0 & 0 & 1 \\
\hline
\end{tabular}

A

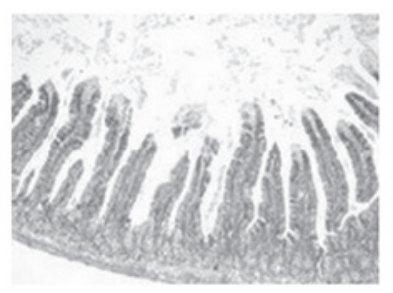

C

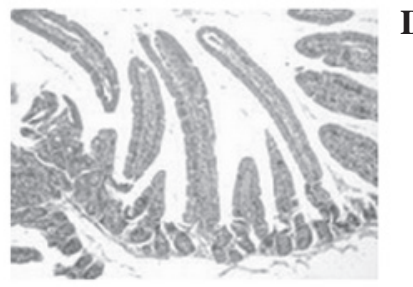

$\mathbf{E}$

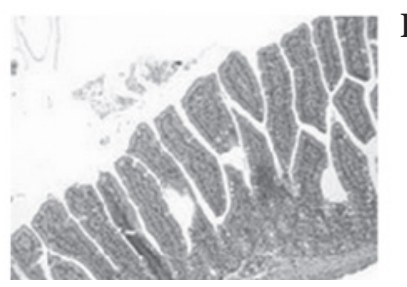

Figure 1. Mouse intestinal tissue with hematoxylin and eosin (H\&E) staining On days $0-9$, the intestinal tissue was complete, with no neutrophilic granulocyte infiltration. On days $0-5$, there were no macrophages in the intestinal tissue; however, a few macrophages were scattered and distributed on days 7 and 9, with no differences between the two groups. (A) Day 0; (B) day 1; (C) day 3; (D) day 5; (E) day 7; (F) day 9. Magnification, $x 40$.

v) steps ii, iii and iv were repeated for 30 cycles for TLR4 mRNA, 35 cycles for NF- $\kappa$ B mRNA and 27 cycles for $\beta$-actin mRNA; vi) $72^{\circ} \mathrm{C} 5 \mathrm{~min}$ for one cycle. The identification of the PCR fragments was confirmed by size following electropho-
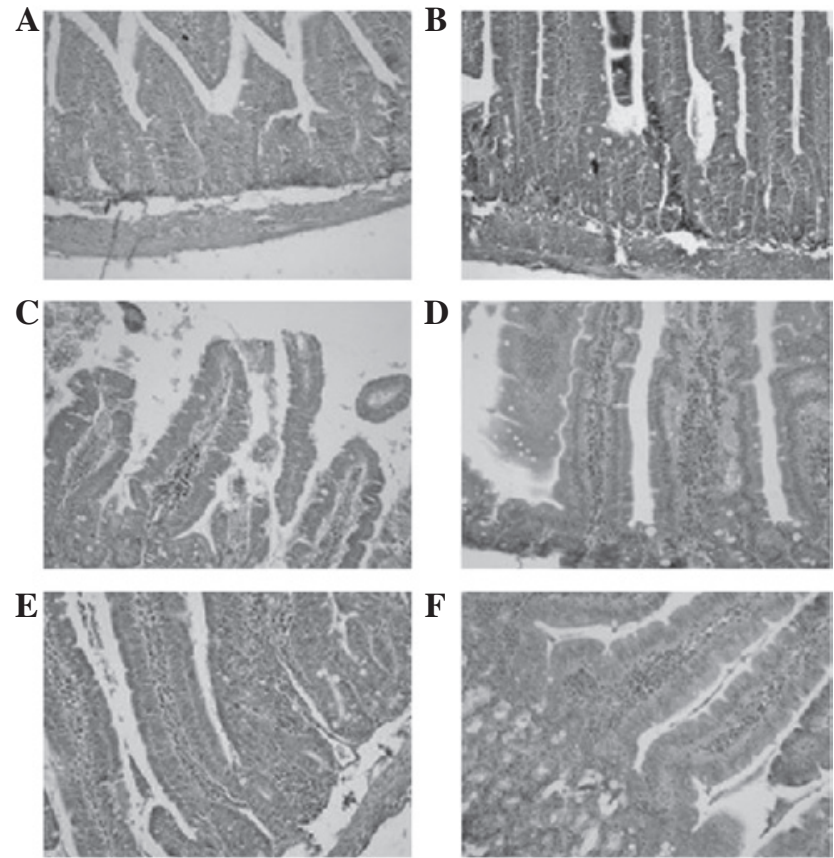

Figure 2. Mouse intestinal tissue with immunohistochemical staining of tumor necrosis factor (TNF)- $\alpha$. On day 0 of high-fat diet stimulation, there were no TNF- $\alpha$-positive cells. However, there were TNF- $\alpha$-positive cells during the following days; a changing trend was observed with time. On day 1 , there were a few cells expressing TNF- $\alpha$ that were continuously distributed in the intestinal epithelium. On day 3 , the TNF- $\alpha$-positive cells in the intestinal epithelium markedly deepened in color, while a few cells began to distribute in the lamina propria mucosa. On day 5 , the TNF- $\alpha$-positive cells in the lamina propria mucosa began to darken in color, increase in number and there was a clustering trend in the distribution. On day 7, the TNF- $\alpha$ positive cells in the lamina propria mucosa were distributed in clusters; however, few were distributed and scattered in the submucosa. On day 9, the TNF- $\alpha$-positive cells were distributed in clusters and dispersed in the lamina propria mucosa and submucosa. (A) Day 0; (B) day 1; (C) day 3; (D) day 5; (E) day 7; (F) day 9. Magnification, x40.

retic migration on ethidium bromide $(0.5 \mathrm{mg} / \mathrm{l})$-stained agarose gels and imaging. The amount of the PCR products was imaged and expressed as optical density. The target cDNA present in each sample was corrected for the respective $\beta$-actin values.

Statistical analysis. Data are presented as mean \pm standard deviation (SD). Student t-tests were performed to determine the statistical significance of protein and mRNA expression levels among the different groups. Enumeration data was analyzed by a rank sum test. $\mathrm{P}<0.05$ was considered to indicate a statistically significant difference.

\section{Results}

$H \&$ E staining. By macroscopic observation, there were no clear changes and no hyperemia or hydrops in the enteric cavity. By light microscopic observation, there was no ulceration or interruption of the intestinal mucosa and no mass neutrophilic granulocyte infiltration. Diffused macrophage distribution was observed on days 7 and 9 (Fig. 1).

Immunohistochemistry. All the groups expressed TNF- $\alpha$ and IL-6, with the exception of the day 0 group. The presence of 

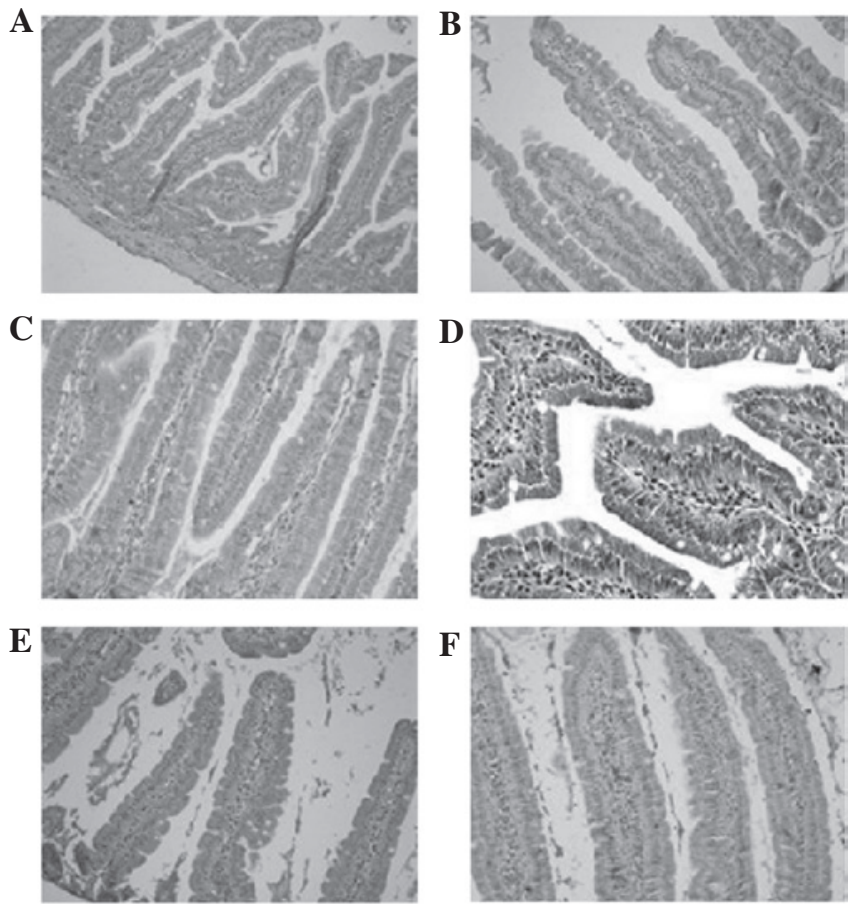

Figure 3. Mouse intestinal IL-6 immunohistochemical staining. On day 0 of high-fat diet stimulation, there were no IL-6-positive cells in the intestines. However, there were IL-6-positive cells during the following days, which gradually changed over time on days 1-9. On day 1, there were a few cells weakly expressing IL-6, which were sporadically distributed in the intestinal epithelium. On day 3, the IL-6-positive cells in the intestinal epithelium were continuously distributed and the color markedly deepened. There were a few pale colored IL-6-positive cells in the lamina propria mucosa. On day 5, the IL-6-positive cells in the lamina propria mucosa darkened in color; however, no changes in quantity or range occurred. On day 7, the amount of IL-6-positive cells in the lamina propria mucosa increased markedly and demonstrated a clustering trend in distribution. On day 9, there were IL-6-positive cells distributed in clusters, dispersed in the lamina propria mucosa but not in the submucosa. (A) Day 0; (B) day 1; (C) day 3; (D) day 5; (E) day 7; (F) day 9. Magnification, $\mathrm{x} 40$.

brown staining in the cytoplasm and/or nuclei indicated that cells were positive for TNF- $\alpha$ and IL-6, which were mainly expressed in the nuclei. We observed that the quantity and distribution of TNF- $\alpha$-positive cells presented dynamic changes with sustained stimulation by a high-fat diet. The expression gradually strengthened and demonstrated cluster distribution in the epithelial mucosa, lamina propria mucosa and submucosa (Table I and Fig. 2). The expression of IL-6positive cells increased gradually in the epithelial mucosa and lamina propria mucosa, and diffused distribution demonstrated a clustering trend (Table II and Fig. 3).

Western blot analysis. On day 0, no TLR4 or NF-кB protein expression was observed in the mouse intestines; however, PIкB protein expression was present and was at its peak level $(\mathrm{P}<0.05)$. At $1,3,5,7$ and 9 days, the TLR4 and NF- $\mathrm{KB}$ protein expression levels increased progressively until day 7 , when peaks were reached $(\mathrm{P}<0.05)$. The expression levels then decreased. The expression levels of PIкB protein decreased gradually and reached a minimum on day $9(\mathrm{P}<0.05$; Fig. 4$)$.

$R T$-PCR. On day 0 , there was no expression of TLR 4 or NF- $\kappa B$ mRNA in the mouse intestines. On days 1, 3, 5, 7 and

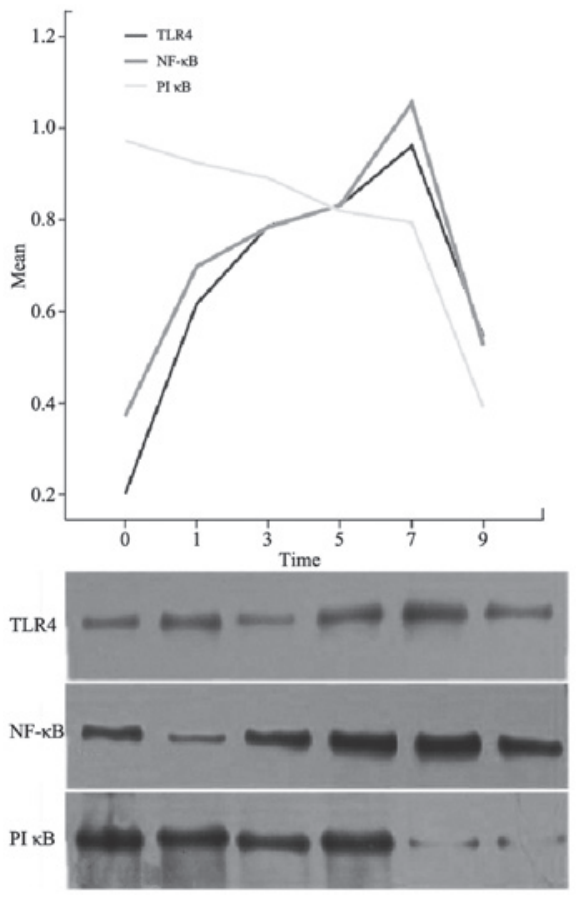

Figure 4. Protein expression of Toll-like receptor (TLR4), NF-кB and PIкB. From day 0 to 1 of high-fat diet stimulation, the expression of TLR4 and NF- $\kappa B$ proteins significantly increased. The protein expression level of TLR4 maintained an increasing trend from day 1 to 7 and the daily rate of increase was less than that on day 1 . The protein expression of NF- $\kappa B$ maintained an increasing trend from day 1 to 7 and the rate of increase on days 5-7 was similar to that on day 1 . The protein expression levels of TLR4 and NF- $\mathrm{KB}$ peaked on day $7(\mathrm{P}<0.05)$, then demonstrated a decreasing trend. The expression of the PIкB protein demonstrated a decreasing trend from day 0 to 9 . The rate of decrease on days 7-9 was significantly different from that on days 0-7, and was the lowest on day $9(\mathrm{P}<0.05)$. The changing trends of TLR4, NF- $\mathrm{KB}$ and PIкB were consistent from day 7 to 9 , with no statistically significant difference in rate $(\mathrm{P}>0.05)$.

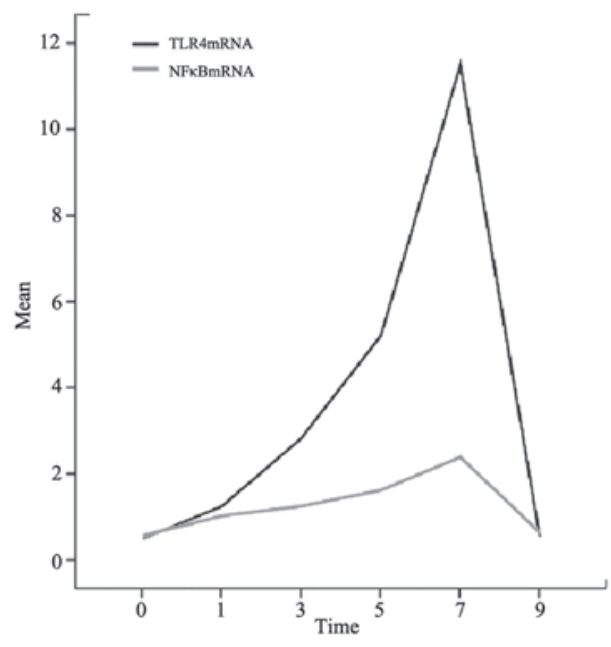

Figure 5. mRNA expression of Toll-like receptor 4 (TLR4) and NF-кB. On day 1 of high-fat diet stimulation, the mRNA expression levels of TLR4 and NF-kB increased; however, the difference between them was not significant $(\mathrm{P}>0.05)$. Then, the expression increased significantly, particularly on days 5-7 and the difference in the expression levels of TLR4 and NF- $\mathrm{\kappa B}$ reached a maximum. On days 5-7, the change in TLR4 mRNA expression was significantly greater than that over the 1-7-day period $(\mathrm{P}<0.05)$. On days $0-5$, the change in NF- $\mathrm{KB}$ mRNA expression was minor and only a slight increase occurred on days 5-7; however, the increase was not statistically significant $(\mathrm{P}>0.05)$. The mRNA expression of TLR4 and NF- $\kappa \mathrm{B}$ peaked on day 7 $(\mathrm{P}<0.05)$, then began to decline; however, the downtrend trend of the expression of TLR4 mRNA was more marked than that of NF- $\mathrm{kB}$ mRNA $(\mathrm{P}<0.05)$. 
9, the mRNA expression levels of TLR4 and NF- $\kappa$ B increased gradually until day 7 , when maximum levels were reached $(\mathrm{P}<0.05)$. Then, a gradual reduction was observed. As a reference, $\beta$-actin expression remained relatively stable (Fig. 5).

\section{Discussion}

Our results show that a short-term, high-fat diet induces local inflammation in mouse intestine, accompanied by activation of TLR4/NF- $\kappa \mathrm{B}$ signaling. This indicates that TLR4/NF- $\kappa \mathrm{B}$ signaling is related to the induction of local inflammation in the intestines caused by a short-term, high-fat diet. The results revealed no clear changes in the physiological structure of intestinal tissue following stimulation with a short-term highfat diet. From day 1 to 9, the intestinal mucosa was complete and a large accumulation of macrophages was not detected. On days 7 and 9, we observed a distribution of macrophages, indicating that the inflammatory reaction shown in this study is different from pathological damage caused by diseases such as ulcerative colitis, since it is low-grade inflammation.

Cani et al (16) hypothesized that bacterial LPS acts as a triggering factor, linking inflammation to high-fat diet-induced diabetes and obesity. The authors identified that consumption of a high-fat diet resulted in significant modulation of the dominant bacterial populations within the gut microflora. Additionally, the authors observed a reduction in the number of bifidobacteria, Eubacterium rectale-Clostridium coccoides species and bacteroides, which favored an increase in the gram-negative to gram-positive ratio. However, in the present study, the observation cycle was shorter than the cycle for bacterial enhancement and LPS release, thus avoiding the interference of LPS generated by intestinal gram-negative bacteria. The results of the present study indicated that a high-fat diet, as an endogenous TLR4 ligand, causes increasing intestinal TLR4 expression. After 1 day of high-fat diet, there was mild activation of intestinal TLR4/NF- $\mathrm{B}$, which increased gradually, peaking on day 7. According to the analysis of the results, the expression of TLR4 and NF- $\kappa \mathrm{B}$ was coincident with the production of TNF- $\alpha$ and IL- 6 . This is in agreement with the results of the study by Tsujimoto et al (17), which demonstrated that the amount of TLR4 expressed was related to the quantity of the inflammatory factor released. PI $\mathrm{B}$, formed by the phosphorylation of $\mathrm{I} \kappa \mathrm{B}$, is the key step for activating the TLR4/NF- $\kappa \mathrm{B}$ signaling pathway. With the continual consumption of intracellular PI $\mathrm{B}$, the activation level of TLR4/NF- $\kappa \mathrm{B}$ increased. After 7 days, the expression of TLR4/NF- $\kappa \mathrm{B}$ began to decrease. This may signify that PIкB was almost exhausted or a protection mechanism was activated.

The local intestinal inflammatory response and inflammatory factors complement each other. Ou et al (18) investigated the expression of TLR4 on human mononuclear/macrophage (THP 1) cell surfaces with flow cytometry. The results demonstrated that the expression of TLR4 on the cell surface may be significantly activated within $24 \mathrm{~h}$ after stimulation by IL-6. Abreu et al (19) identified that activated NF- $\kappa \mathrm{B}$ induces the transcription of TNF- $\alpha$; TNF- $\alpha$ further promotes the expression of NF- $\kappa$ B. Studies have demonstrated that TNF- $\alpha$ induces the apoptosis of intestinal epithelium cells (20-22), as well as a change in the structure and function of tight junction proteins between cells (23), which leads to increased intestinal epithe- lium permeability (24) and eventually causes diffusion of the local inflammatory reaction. Inflammatory agents in the local intestinal reaction, including TNF- $\alpha$ and IL-6, may accelerate the activation of the TLR4/NF- $\kappa \mathrm{B}$ pathway. This may explain why in the current study the activation of the TLR4/NF- $\kappa$ B pathway demonstrated an increasing trend with time.

Reactive oxygen species (ROS) produced as a result of the activation of TLRs, induce a change in cells. Ko et al identified that activation of TLRs in the innate immune response causes retinal photoreceptor oxidative stress and mitochondrial DNA (mtDNA) damage (25). Ye et al (26) expanded our knowledge of TLR4 in a well-characterized mouse model of fatty liver disease induced by a westernized diet. The authors identified that a genetic deletion model of TLR4 (Apoe $\mathrm{ALR}^{-/ /} \mathrm{TLR}^{-/}$) led to a reduction in high-fat, high-cholesterol diet-induced liver inflammation and injury compared with that observed in wild-type mice $\left(\right.$ Apoe $^{-/} / \mathrm{TLR}^{+/+}$), which is associated with the reduced expression of ROS and pro-inflammatory cytokines.

The intestine is the body's largest 'bank of bacteria and endotoxins' with a mucous membrane barrier that is highly selective and maintains normal intestinal physiological activities. The intestinal mucous membrane barrier is chiefly composed of mechanical, immunological and biological barriers. The mechanical barrier is primarily constructed of epithelial cells with a tight junction between them; it is the first line of defense against antigens and toxins from outside. TLR4, which is well distributed on the surface, is able to identify pathogens quickly and rapidly induces an immune inflammatory response. The role of macrophages in local intestinal inflammation should not be ignored. The intestinal immune barrier is mainly constructed of secretory immunoglobulin (SIgA) and SIgA is made up of poly-immunoglobulin (pIgA) and pIgA receptors (pIgR). Since there are a number of different types of glycosyls that are significant bacterial ligands on pIgR, the enteric cavity avoids attack by LPS and enjoys immune protection (27). Multiple factors impact the expression of pIgR, including the nutritional state, as well as cytokines, such as TNF- $\alpha$ (28). An increased level of TNF- $\alpha$ induces enterocytes to secrete more $\mathrm{pIgR}$ at the protein and molecular level, and to increase intestinal immunoprotection. Furthermore, TNF- $\alpha$ also combines with cell surface receptors, including lymphatic toxin receptors and B-cell activators, to participate in the activation of lymphoid organ genes and enable the transcription of the pIgR gene. This is extremely important in limiting the intestinal inflammation caused by viruses and bacteria and in promoting tissue repair (29). It may explain why the protein and mRNA levels of TLR4 and $\mathrm{NF}-\kappa \mathrm{B}$ were markedly reduced on day 9 compared with their levels on day 7.

The hypothesis that TLR4 signaling is involved in autoimmune diseases has prompted research into TLR4 inhibition. One study demonstrated that the binding of mAbs to distinct regions on TLR4 inhibits LPS-dependent activation, providing a novel method for manipulating TLR4 activation and also a rationale for designing drugs targeted to TLR4 (30).

As mentioned previously, we successfully established a model of local intestinal inflammation using a high-fat diet. The digested products of the high-fat diet acted as ligands to rapidly activate the intestinal TLR4/NF- $\kappa \mathrm{B}$ pathway and cause local inflammation. Whether the local intestinal 
low-grade inflammation induced by high-fat diet is a prologue to a systemic inflammation response or a partial expression of systemic inflammation remains unknown and requires further study.

\section{Acknowledgements}

This study was supported by grants from the National Natural Science Foundation of China (30801019) and was also supported by the Department of Metabolic Diseases, VIP Laboratory, First Affiliated Hospital of Xinjiang Medical University.

\section{References}

1. Pickup JC: Inflammation and activated innate immunity in the pathogenesis of type 2 diabetes. Diabetes Care 27: 813-823, 2004

2. Michelsen KS, Doherty TM, Shah PK and Arditi M: TLR signaling: an emerging bridge from innate immunity to atherogenesis. J Immunol 173: 5901-5907, 2004.

3. Kim F, Pham M, Luttrell I, et al: Toll-like receptor-4 mediates vascular inflammation and insulin resistance in diet-induced obesity. Circ Res 100: 1589-1596, 2007.

4. Kopp A, Buechler C, Neumeier M, et al: Innate immunity and adipocyte function: ligand-specific activation of multiple Toll-like receptors modulates cytokine, adipokine, and chemokine secretion in adipocytes. Obesity (Silver Spring) 17: 648-656, 2009.

5. Nguyen MT, Favelyukis S, Nguyen AK, et al: A subpopulation of macrophages infiltrates hypertrophic adipose tissue and is activated by free fatty acids via Toll-like receptors 2 and 4 and JNK-dependent pathways. J Biol Chem 282: 35279-35292, 2007.

6. Song MJ, Kim KH, Yoon JM and Kim JB: Activation of Toll-like receptor 4 is associated with insulin resistance in adipocytes. Biochem Biophys Res Commun 346: 739-745, 2006.

7. Tsukumo DM, Carvalho-Filho MA, Carvalheira JB, et al: Loss-of-function mutation in Toll-like receptor 4 prevents diet-induced obesity and insulin resistance. Diabetes 56: 1986-1998, 2007.

8. Xu H, Barnes GT, Yang Q, et al: Chronic inflammation in fat plays a crucial role in the development of obesity-related insulin resistance. J Clin Invest 112: 1821-1830, 2003.

9. Dasu MR, Devaraj S, Zhao L, Hwang DH and Jialal I: High glucose induces Toll-like receptor expression in human monocytes. Diabetes 57: 3090-3098, 2008.

10. Smiley ST, King JA and Hancock WW: Fibrinogen stimulates macrophage chemokine secretion through toll-like receptor 4. J Immunol 167: 2887-2894, 2001.

11. Arciszewski MB, Sand E and Ekblad E: Vasoactive intestinal peptide rescues cultured rat myenteric neurons from lipopolysaccharide induced cell death. Regul Pept 146: 218-223, 2008.

12. Shi H, Kokoeva MV, Inouye K, Tzameli I, Yin H and Flier JS: TLR4 links innate immunity and fatty acid induced insulin resistance. J Clin Invest 116: 3015-3025, 2006.

13. Oliveira AG, Carvalho BM, Tobar N, et al: Physical exercise reduces circulating lipopolysaccharide and TLR4 activation and improves insulin signaling in tissues of DIO rats. Diabetes 60: 784-796, 2011.
14. Grigoryev DN, Finigan JH, Hassoun P and Garcia JG: Science review: searching for gene candidate in acute lung injury. Crit Care 8: 440-447, 2004.

15. Tschöp M and Thomas G: Fat fuels insulin resistance through Toll-like receptors. Nat Med 12: 1359-1361, 2006.

16. Cani PD, Bibiloni R, Knauf C, et al: Changes in gut microbiota control metabolic endotoxemia-induced inflammation in high-fat diet-induced obesity and diabetes in mice. Diabetes 57 : 1470-1481, 2008.

17. Tsujimoto H, Ono S, Efron PA, Scumpia PO, Moldawer LL and Mochizuki H: Role of Toll-like receptors in the development of sepsis. Shock 29: 315-321, 2008.

18. Ou HQ, Ma XH, Shen J and Shen HJ: Effects of human interleukin-6 (IL-6) on the expression of Toll like receptor-4 (TLR4) of THP-1. Acta Universitatis Medicinalis Nanjing (Natural Science) 30: 319-323, 2010.

19. Abreu MT, Vora P, Fature E, Thomas LS, Arnold ET and Arditi M: Decreased expression of Toll-like receptor- 4 and MD-2 correlates with intestinal epithelial cell protection against dysregulated proinflammatory gene expression in response to bacterial lipopolysaccharide. J Immunol 167: 1609-1616, 2001.

20. Bojarski C, Bendfeldt K, Gitter AH, et al: Apoptosis and intestinal barrier function. Ann NY Acad Sci 915: 270-274, 2000.

21. Bruewer M, Luegering A, Kucharzik T, et al: Proinflammatory cytokines disrupt epithelial barrier function by apoptosis-independent mechanisms. J Immunol 171: 6164-6172, 2003.

22. Pinkoski MJ, Droin NM and Green DR: Tumor necrosis factor alpha up-regulates non-lymphoid Fas-ligand following superantigen-induced peripheral lymphocyte activation. J Biol Chem 277: 42380-42385, 2002.

23. Poritz LS, Garver KI, Tilberg AF and Koltun WA: Tumor necrosis factor alpha disrupts tight junction assembly. J Surg Res 116: 14-18, 2004.

24. Ma TY, Iwamoto GK, Hoa NT, et al: TNF-alpha induced increase in intestinal epithelial tight junction permeability requires NF-kappa B activation. Am J Physiol Gastrointest Liver Physiol 286: G367-G376, 2004.

25. Ko MK, Saraswathy S, Parikh JG and Rao NA: The role of TLR4 activation in photoreceptor mitochondrial oxidative stress. Invest Ophthalmol Vis Sci 52: 5824-5835, 2011.

26. Ye D, Li FY, Lam KS, et al: Toll-like receptor-4 mediates obesity-induced non-alcoholic steatohepatitis through activation of X-box binding protein-1 in mice. Gut 61: 1058-1067, 2012.

27. Bruno ME and Kaetzel CS: Long-term exposure of the HT-29 human intestinal epithelial cell line to TNF causes sustained up-regulation of the polymeric Ig receptor and pro-inflammatory genes through transcriptional and posttranscriptional mechanisms. J Immunol 174: 7278-7284, 2005.

28. Schjerven H, Tran TN, Brandtzaeg P and Johansen FE: De novo synthesized RelB mediates TNF-induced up-regulation of the human polymeric Ig receptor. J Immunol 173: 1849-1857, 2004.

29. Murthy AK, Dubose CN, Banas JA, Coalson JJ and Arulanandam BP: Contribution of polymeric immunoglobulin receptor to regulation of intestinal inflammation in dextran sulfate sodium induced colitis. J Gastroenterol Hepatol 21: 1372-1380, 2006.

30. Tsukamoto H, Fukudome K, Takao S, et al: Multiple potential regulatory sites of TLR4 activation induced by LPS as revealed by novel inhibitory human TLR4 mAbs. Int Immunol 24: 495-506, 2012. 